\title{
Glass Ionomer Sealant versus Fluoride Varnish Application to Prevent Occlusal Caries in Primary Second Molars among Preschool Children: A Randomized Controlled Trial
}

\author{
Phoebe Pui Ying Lama ${ }^{a}$ Divesh Sardana ${ }^{a}$ Weijia Luo ${ }^{a}$ \\ Manikandan Ekambaram ${ }^{b}$ Gillian Hiu Man Lee ${ }^{a}$ Edward Chin Man Lo ${ }^{c}$ \\ Cynthia Kar Yung Yiu ${ }^{a}$ \\ aPaediatric Dentistry, Faculty of Dentistry, The University of Hong Kong, Hong Kong, Hong Kong SAR; bPaediatric \\ Dentistry, Faculty of Dentistry, University of Otago, Dunedin, New Zealand; 'Dental Public Health, Faculty of \\ Dentistry, The University of Hong Kong, Hong Kong, Hong Kong SAR
}

\section{Keywords}

Pit and fissure sealants - Fluoride varnish - Caries prevention .

Preschool children $\cdot$ Randomized controlled trial

\begin{abstract}
Fissure sealant is a recommended preventive measure on permanent molars, but its efficacy on primary molars in preschool children is still in doubt. Sodium fluoride varnish $(\mathrm{NaFV})$ enhances remineralization and is effective in preventing caries in smooth surfaces, but limited information is available regarding its use on occlusal surfaces of primary molars. The present study aimed to compare the efficacy of glass ionomer sealant (GIS) versus topical application of $5 \%$ $\mathrm{NaFV}$ in preventing and arresting occlusal caries in primary second molars among preschool children. One calibrated examiner examined children aged 3-4 years for visible plaque index (VPI), decayed-missing-filled primary surface (dmfs) index, and extent of carious lesions by the International Caries Detection and Assessment System (ICDAS). Subjects with 1 or more primary second molars that were sound or with incipient lesions (ICDAS 0-1), with distinct visual enamel changes (ICDAS 2), or with localized enamel
\end{abstract}

karger@karger.com www.karger.com/cre

Karger $\stackrel{\text { ' }}{5}$
(C) 2021 The Author(s)

Published by S. Karger AG, Basel

This is an Open Access article licensed under the Creative Commons Attribution-NonCommercial-4.0 International License (CC BY-NC) (http://www.karger.com/Services/OpenAccessLicense), applicable to the online version of the article only. Usage and distribution for commercial purposes requires written permission. breakdown (ICDAS 3) were recruited. The children were randomly assigned to either NaFV group - application of $5 \%$ $\mathrm{NaFV}$ at 3-monthly intervals or GIS group - 1 single placement of GIS. A parental questionnaire was used to collect information on the children's sociodemographic background and oral health-related habits. Two blinded examiners conducted clinical examinations after 6 and 12 months to evaluate the development of occlusal caries and the retention of GIS. Negative binomial regression using generalized estimating equations was employed to adjust for the clustering effect and evaluate the influence of selected variables on the development of occlusal caries into dentin at 12 months. A total of 323 children with 1,159 primary second molars received the respective preventive measures at baseline. At 12 months, 280 children with 989 molars were evaluated. Caries had developed or progressed into dentin in $7.8 \%$ and $8.0 \%$ of the molars in the NaFV and GIS groups, respectively, with no significant difference between the groups ( $p=0.913)$. The overall retention rates of GIS were $24.6 \%$ and $13.5 \%$ at 6 and 12 months, respectively. Regression analyses showed only baseline caries experience, and an ICDAS code had a significant association with occlusal caries progression. Quarterly NaFV application and single 
GIS placement showed similar effectiveness in the prevention of occlusal caries development among preschool children.

() 2021 The Author(s).

Published by S. Karger AG, Basel hypothesis tested was that there is no difference between the placement of GIS and the topical application of $5 \%$ $\mathrm{NaFV}$ in the prevention of occlusal caries in primary second molars among preschool children.

\section{Subjects, Materials, and Methods}

This parallel-group randomized controlled trial was conducted in Hong Kong, where the communal water supply is fluoridated optimally at a concentration of $0.5 \mathrm{ppm}$. Most child formula toothpastes available for purchase in Hong Kong contain fluoride at a concentration of 500-600 ppm. Ethical approval was obtained from the Institutional Review Board of the University of Hong Kong/Hospital Authority Hong Kong West Cluster (UW 18-053), and the trial was registered in ClinicalTrials.gov (NCT04163354).

\section{Sample Size Calculation}

The primary outcome measure of this clinical trial was caries development into dentin on the occlusal surface of primary second molar, which was assessed by the International Caries Detection and Assessment System (ICDAS) II codes 4, 5, and $6\left[\left(^{*}\right)\right]$. Based on the results of a previous split-mouth study on primary molars $[(*)]$, the absolute difference between the effect of fluoride varnish and that of sealant on occlusal caries reduction was 30\%. Calculated with a $5 \%$ statistical significance level and a $90 \%$ power, a minimum of 102 children in each group were required. The initial sample size was increased by $25 \%$ to allow for loss of power due to the dropout of study participants, that is, 128 children in each group. Thus, the total sample size needed at the beginning of the study was 256 divided into 2 equal groups.

\section{Subject Selection}

Children attending kindergarten grade 1 in 11 kindergartens dispersed across Hong Kong were invited to participate in the present study. An invitation letter was sent to their parents, explaining the objectives and procedures of the study. Only children whose parents signed the informed consent were included. Parents were also required to complete a validated questionnaire regarding their child's socioeconomic background and oral health-related behaviors.

\section{Inclusion Criteria}

After screening, children deemed to be at moderate to high caries risk, that is, who fulfilled at least one of the following criteria as defined in the guidelines provided by the American Academy of Pediatric Dentistry $[(*)]$ were identified. The criteria were children who were recent immigrants or of low socioeconomic status, snacked $>3$ times per day, or were put to bed with a bottle containing natural or added sugar, as well as children with visible plaque, $>1$ decayed-missing-filled tooth surfaces, white spot lesions, or enamel defects $\left.\left[{ }^{*}\right)\right]$.

\section{Exclusion Criteria}

According to the self-reported questionnaire, children with serious systemic disease or who required long-term medication, as well as children with special health-care needs were excluded. Also, children who were uncooperative during the interventions or who had received professional topical fluoride application in the past 6 
months were excluded from the study. Primary second molars with restorations, sealants, or dentinal caries being assessed as ICDAS II code 4,5 , or $6\left[\left(^{*}\right)\right]$ were excluded. The study also excluded molars that were partially erupted, hypoplastic, or hypomineralized.

\section{Subject Recruitment}

Subject recruitment lasted from April until July in 2018. A total of 367 parental written consents were obtained from children attending kindergarten grade 1 in the 11 study kindergartens. Clinical examination was performed on 356 children as 11 children were either absent on the day of visit (2 children) or refused to be examined ( 9 children). Since all children examined were presented with visible plaques, all invited children were assessed as of moderate caries risk or above and thereby met the inclusion criteria. Twenty-three children were further excluded due to the presence of caries in all 4 primary second molars with ICDAS code 4 or above (16 children), the presence of restorations on all primary second molars ( 1 child), age over 60 months ( 4 children), and complex systemic health conditions ( 2 children). After the clinical examination, 154 children with at least 1 primary second molar with ICDAS codes $0-3$ were assigned to the NaFV group, and all of them received the application of NaFV. On the other hand, 179 children were allocated to the GIS group, but GIS was only able to be placed on at least one of the second primary molars of 169 children. Seven children were not cooperative during the clinical procedures, while another 3 children had strong gag reflex that precluded GIS placement. The mean age of the NaFV group children was $46.5( \pm 3.7)$ months while that of the GIS group children was $46.3( \pm 3.7)$ months. The respective total numbers of second primary molars in the NaFV and GIS groups were 567 and 592.

\section{Questionnaire}

A validated parental questionnaire, which was adapted from earlier studies $\left[\left(^{*}\right)\right]$, was distributed to collect the children's personal data (gender, date and place of birth, medical history of systemic diseases, and long-term medication); oral health-related behaviors (toothbrushing frequency, supervised toothbrushing, use of toothpaste, snacking habit, night bottle feeding, and frequency of dental visits); and socioeconomic background (parents' age and education, monthly household income, and the number of siblings).

\section{Clinical Examination}

Clinical examination was performed by 1 dentist (P.L.), with the child lying supine on a table provided by the kindergarten, using disposable dental mirrors attached to an intraoral light-emitting diode (MirrorLite, Kudos Crown Ltd., Hong Kong) and a blunt WHO CPI probe. The visible plaque index (VPI) $\left[\left(^{*}\right)\right]$ score and the number of decayed-missing-filled primary teeth $(\mathrm{dmft})$ and decayed-missing-filled primary surfaces $(\mathrm{dmfs})\left[\left(^{*}\right)\right]$ were recorded. After cleaning and drying the tooth surfaces with gauze, the caries status of the occlusal surfaces of primary second molars was recorded using the ICDAS II coding system. As air drying was not possible in outreach settings, ICDAS 0 and 1 lesions were recorded in combination to enhance detection reliability. The ball end of a CPI probe was used to slide through the pit and fissures to detect any enamel cavities or discontinuities $\left[\left(^{*}\right)\right]$. A $10 \%$ random sample was reexamined during every examination to monitor intra-examiner reproducibility.

\section{Randomization and Treatment Allocation}

The recruited children were randomly allocated using sequentially numbered, opaque, and sealed envelopes to either the NaFV group or the GIS group. The random allocation was concealed as the envelopes were opened only in front of the participants, just before their respective allocation, following a computer-generated list of random numbers.

\section{Interventions}

For the sealant group, GIS was applied using the finger-press technique described in the WHO manual for atraumatic restorative treatment $\left[\left(^{*}\right)\right]$ by the same dentist (P.L.) after randomization. The primary second molars that were included were first cleaned and dried by wiping with gauze and cotton pellets. Following the manufacturer's instructions, further cleaning of the fissures was performed by applying GC cavity conditioner with a microapplicator for $10-15 \mathrm{~s}$ and then cleaned with wet cotton pellets for several times. The surface was dried with cotton pellets. To standardize the liquid-powder ratio of the GIC (GC Fuji VII, GC Asia), capsules were used and mixed with an amalgamator, followed by its application on the occlusal surface with a plastic instrument, and were slightly overfilled. A gloved finger with petroleum jelly (Vaseline ${ }^{\circledR}$ ) was used to place and rub the materials into the pits and fissures, as well as to remove the excess GIC material from the tooth surface. The operator followed a standard protocol for each included child and first performed the procedure on tooth 85 , followed by teeth 75,65 , and finally on 55 .

In the NaFV group, $0.25 \mathrm{~mL}$ (1 drop) of the varnish (Colgate Duraphat $^{\circledR}$ varnish, Colgate-Palmolive [UK] Ltd., $50 \mathrm{mg} / \mathrm{mL}$ ) was placed in a plastic dappen dish by the same dentist (P.L.). A disposable microbrush was used to apply the varnish onto the second primary molars included in the study and all the other teeth. The child was instructed not to eat or drink after the application of varnish for at least half an hour.

\section{Follow-Up Evaluation}

The same dentist (P.L.) visited the kindergartens every 3 months to apply NaFV on the study teeth of the NaFV group children. Similar to the baseline, the same amount of $5 \% \mathrm{NaFV}$ was applied using a disposable microbrush. At 6 and 12 months, a clinical examination was performed by 2 other examiners (D.S. and W.L.) for all children in both groups. During the follow-up examination, overall VPI and dmfs were recorded. ICDAS scores were recorded for each primary second molar included in the study.

\section{Outcome Measures}

The primary outcome measure was occlusal caries development into dentin (ICDAS codes 4,5 , and 6 ) in the primary second molars. The secondary outcome measure was the retention of GIS.

\section{Data Processing and Analysis}

Data analysis was performed using SPSS ${ }^{\circledR}$ Statistics, version 23.0 (SPSS Inc, Chicago, IL, USA). Children with missing outcome data resulting from loss to follow-up or unrecorded data were excluded from the analysis. Data proofreading was performed after data entry to identify and correct any errors before analyzing the data. An intention-to-treat analysis was undertaken. Intra- and inter-examiner agreement in diagnosing the occurrence and severity of dental caries were assessed by Cohen's kappa statistics. The 




Fig. 1. Flowchart of participants through each stage of randomized controlled trial. ICDAS, International Caries Detection and Assessment System; NaF varnish, sodium fluoride varnish; GIS, glass ionomer sealant. 
Table 1. Baseline demographic profile and oral health status of study participants $(n=323)$

\begin{tabular}{|c|c|c|c|}
\hline Child demographic profile & $\begin{array}{l}\text { NaFV group }(n=154) \\
\%(n)\end{array}$ & $\begin{array}{l}\text { GIS group }(n=169) \\
\%(n)\end{array}$ & $p$ value \\
\hline \multicolumn{4}{|l|}{ Child's gender } \\
\hline Male & $49.4(76)$ & $56.8(96)$ & \multirow{2}{*}{$0.180^{\dagger}$} \\
\hline Female & $50.6(78)$ & $43.2(73)$ & \\
\hline \multicolumn{4}{|l|}{ Place of birth } \\
\hline Hong Kong & $89.6(138)$ & $93.5(158)$ & \multirow{2}{*}{$0.208^{\dagger}$} \\
\hline Others or undisclosed & $10.4(16)$ & $6.5(11)$ & \\
\hline \multicolumn{4}{|l|}{ Parent age range } \\
\hline $20-29$ years & $10.4(16)$ & $10.1(17)$ & \multirow{5}{*}{$0.739^{\ddagger}$} \\
\hline $30-39$ years & $66.9(103)$ & $65.7(111)$ & \\
\hline $40-49$ years & $19.5(30)$ & $22.5(38)$ & \\
\hline 50 years or above & $1.9(3)$ & $1.8(3)$ & \\
\hline Undisclosed & $1.3(2)$ & $0.0(0)$ & \\
\hline \multicolumn{4}{|l|}{ Family income, HKD per month } \\
\hline$<$ USD 20,000 & $34.4(53)$ & $37.3(63)$ & \multirow{5}{*}{$0.948^{\dagger}$} \\
\hline USD $20,001-30,000$ & $20.1(31)$ & $19.5(33)$ & \\
\hline USD $30,001-40,000$ & $14.3(22)$ & $13.0(22)$ & \\
\hline USD 40,000 or above & $21.4(33)$ & $22.5(38)$ & \\
\hline Undisclosed & $9.7(15)$ & $7.7(13)$ & \\
\hline \multicolumn{4}{|l|}{ Father's education level } \\
\hline Less than primary & $1.3(2)$ & $0.6(1)$ & \multirow{5}{*}{$0.258^{\dagger}$} \\
\hline Secondary school & $51.2(79)$ & $46.7(79)$ & \\
\hline Post-secondary/university & $24.7(38)$ & $35.5(60)$ & \\
\hline Postgraduate or above & $13.6(21)$ & $9.5(16)$ & \\
\hline Undisclosed & $9.1(14)$ & $7.7(13)$ & \\
\hline \multicolumn{4}{|l|}{ Mother's education level } \\
\hline Less than primary & $4.5(7)$ & $3.0(5)$ & \multirow{5}{*}{$0.765^{\dagger}$} \\
\hline Secondary school & $55.2(85)$ & $53.8(91)$ & \\
\hline Post-secondary/university & $29.9(46)$ & $33.7(57)$ & \\
\hline Post-graduate or above & $9.1(14)$ & $7.1(12)$ & \\
\hline Undisclosed & $1.3(2)$ & $2.4(4)$ & \\
\hline \multicolumn{4}{|l|}{ Children, $n$} \\
\hline 1 & $31.2(48)$ & $36.1(61)$ & \multirow{4}{*}{$0.760^{\dagger}$} \\
\hline 2 & $56.5(87)$ & $50.9(86)$ & \\
\hline$<3$ & $8.4(13)$ & $9.5(16)$ & \\
\hline \multirow[t]{2}{*}{ Undisclosed } & $3.9(6)$ & $3.6(6)$ & \\
\hline & Mean (SD) & Mean SD & $p$ value \\
\hline Age, months & $46.5(3.7)$ & $46.3(3.7)$ & $0.724^{\S}$ \\
\hline Oral habits & $\%(n)$ & $\%(n)$ & $p$ value $^{\dagger}$ \\
\hline \multicolumn{4}{|l|}{ Brushing frequency } \\
\hline Less than once a day & $9.7(15)$ & $7.7(13)$ & \multirow{4}{*}{$0.865^{\ddagger}$} \\
\hline Once a day & $32.5(50)$ & $32.5(55)$ & \\
\hline Twice or more a day & $57.8(89)$ & $59.2(96)$ & \\
\hline Undisclosed & $0.0(0)$ & $0.6(1)$ & \\
\hline \multicolumn{4}{|l|}{ Supervised toothbrush } \\
\hline No & $9.7(15)$ & $7.7(13)$ & \multirow{4}{*}{$0.374^{\ddagger}$} \\
\hline Yes, sometimes & $36.4(56)$ & $45.6(77)$ & \\
\hline Yes, all the time & $52.6(81)$ & $46.2(78)$ & \\
\hline Undisclosed & $1.3(2)$ & $0.6(1)$ & \\
\hline
\end{tabular}


Table 1 (continued)

\begin{tabular}{|c|c|c|c|}
\hline Oral habits & $\%(n)$ & $\%(n)$ & $p$ value $^{\dagger}$ \\
\hline \multicolumn{4}{|l|}{ Toothpaste } \\
\hline No toothpaste & $9.1(14)$ & $5.9(10)$ & \multirow{5}{*}{$0.387^{\dagger}$} \\
\hline Child nonfluoridated toothpaste & $36.4(56)$ & $32.5(55)$ & \\
\hline Child or adult fluoridated toothpaste & $41.6(64)$ & $42.6(72)$ & \\
\hline Uncertain & $11.0(17)$ & $17.8(30)$ & \\
\hline Undisclosed & $1.9(3)$ & $1.2(2)$ & \\
\hline \multicolumn{4}{|l|}{ Snacking frequency } \\
\hline Less than once a day & $11.0(17)$ & $12.4(19)$ & \multirow{5}{*}{$0.276^{\dagger}$} \\
\hline Once a day & $33.8(52)$ & $23.7(40)$ & \\
\hline Twice a day & $35.1(56)$ & $42.6(72)$ & \\
\hline Over twice & $20.1(31)$ & $20.7(35)$ & \\
\hline Undisclosed & $0.0(0)$ & $1(0.6)$ & \\
\hline \multicolumn{4}{|l|}{ Night bottle habit } \\
\hline Yes & $11.0(17)$ & $11.2(19)$ & \multirow{4}{*}{$0.881^{*}$} \\
\hline Previously yes but winded up recently & $29.9(46)$ & $30.2(51)$ & \\
\hline Never & $58.4(90)$ & $56.8(96)$ & \\
\hline Undisclosed & $0.6(1)$ & $1.8(3)$ & \\
\hline \multicolumn{4}{|l|}{ Previous dental visit } \\
\hline No & $83.8(129)$ & $88.32(149)$ & \multirow{2}{*}{$0.254^{\dagger}$} \\
\hline Yes & $16.2(25)$ & $11.8(20)$ & \\
\hline Oral health parameters & Mean (SD) & Mean (SD) & $p$ value \\
\hline VPI score (\%) & $32.5(19.3)$ & $30.2(18.2)$ & $0.253^{\S}$ \\
\hline dmft score & $1.2(2.7)$ & $1.2(2.5)$ & $0.977^{\S}$ \\
\hline dt score & $1.2(2.7)$ & $1.2(2.5)$ & $0.978^{\S}$ \\
\hline $\mathrm{ft}$ score & $0.0(0.1)$ & $0.0(0.1)$ & $0.948^{\S}$ \\
\hline mt score & $0.0(0.0)$ & $0.0(0.0)$ & $\mathrm{N} / \mathrm{A}$ \\
\hline dmfs score & $1.8(4.2)$ & $2.0(4.4)$ & $0.686^{\S}$ \\
\hline ds score & $1.8(4.2)$ & $2.0(4.4)$ & $0.712^{\S}$ \\
\hline fs score & $0.0(0.1)$ & $0.0(0.3)$ & $0.502^{\S}$ \\
\hline ms score & $0.0(0.0)$ & $0.0(0.0)$ & N/A \\
\hline
\end{tabular}

VPI, visible plaque index; NaFV, sodium fluoride varnish; GIS, glass ionomer sealant. Percentage of surfaces with plaque present on the buccal and palatal/lingual surfaces of 6 index teeth (teeth 55, 51, 63, 71, 75, 83). N/A, not applicable. Cannot be computed because the SD of both groups is zero. ${ }^{\dagger} p$ values derived from $\chi^{2}$ statistics. ${ }^{\ddagger} p$ values derived from Fisher's exact statistics. ${ }^{\S} p$ value derived from $t$-test for independent samples.

$\chi^{2}$ test was used to assess the difference between the 2 groups regarding the distribution of children's demographic characteristics such as gender and place of birth, oral health-related behavior, use of fluoride toothpaste, snacking habit, parents' education, and family income. The outcome measure was the occurrence of new dentinal caries. The $\chi^{2}$ test and multiple logistic regression were employed for within-group comparisons of changes. Student's $t$ test was used to differentiate the comparability between the study groups according to the children's baseline conditions, including age, dmfs score, and VPI score.

Since more than 1 molar from a child was included, generalized estimating equations (GEEs) were used to account for the correlation (clustering) between observations of multiple molars from the same child $\left[\left(^{*}\right)\right]$. The GLIMMIX procedure was used to adjust for the clustering effect as observations from the same child would not be independent $\left.\left[{ }^{*}\right)\right]$. The first and second levels were the primary second molar and the child, respectively. Negative binomial regression using GEE was employed to analyze the contribution of each factor to caries progression at 12 months and hence constructed a prediction model for occlusal caries progression under the influence of the 2 preventive measures $\left[\left(^{*}\right)\right]$. All variables were regrouped and evaluated with GEE, in which the most insignificant variable was removed one by one by a backward stepwise procedure. The process was repeated until all retained variables demonstrated a significant association with the outcome $(p<0.05)$.

\section{Dropout Rate at Follow-Up Examinations}

At 6 months, a total of 288 children with 1,081 molars were evaluated. Thirty-five recruited children with 78 molars could not be assessed as they were absent ( 24 children; 30 molars), withdrew from the study (10 children; 38 molars), or refused examination (1 
child; 4 molars). The remaining 6 molars were excluded as they were restored. No significant difference was found between the dropout rates of the 2 intervention groups $(p=0.805)$.

At 12 months, the dropout rates for the NaFV and GIS groups were $13.6 \%$ and $13.0 \%$, respectively $(p=0.870)$. Two hundred eighty children with 989 molars were evaluated and analyzed. Forty-three children with 170 molars were excluded due to absence ( 23 children; 77 molars), withdrawal (19 children and 74 molars), uncooperativeness for the examination (1 child, 4 molars), and existing fillings (15 molars). The flow of subjects over the 12-month study period is shown in Figure 1.

\section{Intra- and Inter-Examiner Reliability}

All 3 examiners (P.L, D.S., and L.L) received training and had been using the VPI, dmft, and ICDAS examination for over 1 year in the same dental hospital. The kappa value for intra-examiner reliability (PL) for the VPI, dmft, and ICDAS was $0.774,0.964$, and 0.834 , respectively. During the follow-up reviews in similar outreach settings, the inter-examiner reliability for $\mathrm{dmft}$ and ICDAS was 0.902 and 0.700 (P.L. and D. S), as well as 0.899 and 0.785 (P.L and W.L), respectively.

\section{Results}

Demographic Background of the Children Assessed and Those Lost to Follow-Up

No statistically significant difference in the rates of loss to follow-up was found between the NaFV and GIS groups at 12 months $(p=0.870)$. There were also no significant differences in all the evaluated independent variables regarding demographic background, oral health behaviors, and baseline clinical findings between the children lost to follow-up and those followed up at 12 months (see online suppl. material; for all online suppl. material, see www. karger.com/doi/10.1159/000517390).

\section{Demographic Background of Children}

No statistically significant difference was found between the children in the 2 study groups with respect to their demographic background, oral health behaviors, and oral health parameters. Regarding the second primary molars included in the 2 groups, no significant differences were found in terms of caries severity in teeth 55 , 65 , and 75 , but a higher proportion of molars with ICDAS code 1 or 2 was identified in tooth $85(p=0.024)$ and hence the overall primary second molars included $(p=$ 0.003) in the GIS group (Tables 1, 2).

\section{Occlusal Caries Progression at 6 and 12 Months}

At 6 months, only $1.6 \%$ of all included molars $(17 / 1,081)$ had caries progressed into dentin (ICDAS code 4 or higher). The proportion of included molars
Table 2. Baseline ICDAS status of included primary second molars

\begin{tabular}{cccc}
\hline & $\begin{array}{l}\text { NaFV group }\left(N \begin{array}{l}\text { GIS group }(N \\
\text { children }=154) \\
\%(n)\end{array}\right. \\
\text { children }=169) \\
\%(n)\end{array}$ & $p$ value \\
\hline Tooth 55 & $n=145$ & $n=141$ & \\
ICDAS 0-1 & $86.9(126)$ & $87.2(123)$ & \\
ICDAS 2 & $4.1(6)$ & $6.4(9)$ & $0.520^{\dagger}$ \\
ICDAS 3 & $9.0(13)$ & $6.4(9)$ & \\
Tooth 65 & $n=144$ & $n=143$ & \\
ICDAS 0-1 & $91.7(132)$ & $86.7(124)$ & \\
ICDAS 2 & $3.5(5)$ & $7.7(11)$ & $0.278^{\dagger}$ \\
ICDAS 3 & $4.9(7)$ & $5.6(8)$ & \\
Tooth 75 & $n=138$ & $n=151$ & \\
ICDAS 0-1 & $86.2(119)$ & $82.1(124)$ & \\
ICDAS 2 & $6.5(9)$ & $11.3(17)$ & \\
ICDAS 3 & $7.2(10)$ & $6.6(10)$ & \\
Tooth 85 & $n=140$ & $n=159$ & \\
ICDAS 0-1 & $89.3(125)$ & $79.9(127)$ & \\
ICDAS 2 & $4.3(6)$ & $13.2(21)$ & $0.024^{*, \dagger}$ \\
ICDAS 3 & $6.4(9)$ & $6.9(11)$ & \\
All included teeth & $n=567$ & $n=592$ & \\
ICDAS 0-1 & $88.5(502)$ & $84.0(497)$ & \\
ICDAS 2 & $4.6(26)$ & $9.8(58)$ & $0.003^{* *, \dagger}$ \\
ICDAS 3 & $6.9(39)$ & $6.3(37)$ & \\
\hline
\end{tabular}

ICDAS, International Caries Detection and Assessment System; NaFV, sodium fluoride varnish; GIS, glass ionomer sealant. ${ }^{\dagger} p$-values derived from $\chi^{2}$ statistics. ${ }^{*} p<0.05$. ${ }^{* *} p<0.01$.

with caries progression into dentin in the $\mathrm{NaFV}$ and GIS groups were $1.3 \%$ (7/524 molars) and 1.8\% (10/557 molars), respectively ( $p=0.549)$ (Table 3 ).

At 12 months, the overall prevalence of primary second molars with occlusal caries into dentin was $7.9 \%$. No significant difference was found between the 2 study groups (NaFV group, 7.8\%, 37/475 molars; GIS group, $8.0 \%, 41 / 514$ molars; $p=0.913$ ) (Table 3).

\section{Retention Rate of GIS at 6 and 12 Months}

The overall retention rates of GIS at 6 months and 12 months were $24.6 \%(137 / 557)$ and $13.5 \%$ (70/514), respectively. The retention rate at 6 months was the highest for GIS placed on tooth 85 (29.7\%), and the lowest was found on tooth 65 (21.5\%). At 12 months, the retention rate was the highest for tooth $75(17.3 \%)$ and the lowest for tooth 55 (10.8\%). However, the findings were not of statistical significance at both time points (6 months: $p=$ 0.144; 12 months: $p=0.156$ ) (Table 4 ).

\section{Negative Binomial Regression at 12 Months}

Employing the GLIMMIX procedure to adjust for the clustering effect, the regression analysis results showed 
Table 3. Six- and 12-month ICDAS status of included primary second molars

\begin{tabular}{|c|c|c|c|}
\hline & $\begin{array}{l}\text { NaFV group }(N \\
\text { children }=154) \\
\%(n)\end{array}$ & $\begin{array}{l}\text { GIS group }(N \\
\text { children = 169) } \\
\%(n)\end{array}$ & $p$ value \\
\hline \multicolumn{4}{|l|}{6 months } \\
\hline Baseline ICDAS 0-1 & $n=463$ & $n=468$ & \\
\hline$\geq \operatorname{ICDAS} 4$ & $0.0(0)$ & $0.2(1)$ & $0.503^{\ddagger}$ \\
\hline ICDAS 0-3 & $100.0(463)$ & $99.8(467)$ & \\
\hline Baseline ICDAS 2 & $n=25$ & $n=56$ & \\
\hline$\geq \operatorname{ICDAS} 4$ & $8.0(2)$ & $5.4(3)$ & $0.493^{\ddagger}$ \\
\hline ICDAS $0-3$ & $92.0(23)$ & $94.6(53)$ & \\
\hline Baseline ICDAS 3 & $n=36$ & $n=33$ & \\
\hline$\geq \operatorname{ICDAS} 4$ & $13.9(5)$ & $18.2(6)$ & $0.627^{\dagger}$ \\
\hline ICDAS $0-3$ & $86.1(31)$ & $81.8(27)$ & \\
\hline All included teeth & $n=524$ & $n=557$ & \\
\hline$\geq \operatorname{ICDAS} 4$ & $1.3(7)$ & $1.8(10)$ & $0.368^{\dagger}$ \\
\hline ICDAS 0-3 & $98.7(517)$ & $98.2(547)$ & \\
\hline \multicolumn{4}{|l|}{12 months } \\
\hline Baseline ICDAS 0-1 & $n=420$ & $n=431$ & \\
\hline$\geq$ ICDAS 4 & $1.8(15)$ & $1.8(15)$ & $0.943^{\dagger}$ \\
\hline ICDAS 0-3 & $96.4(405)$ & $96.5(416)$ & \\
\hline Baseline ICDAS 2 & $n=23$ & $n=54$ & \\
\hline$\geq \operatorname{ICDAS} 4$ & $22.1(6)$ & $22.2(12)$ & $0.714^{\dagger}$ \\
\hline ICDAS 0-3 & $73.9(17)$ & $77.8(42)$ & \\
\hline Baseline ICDAS 3 & $n=32$ & $n=29$ & \\
\hline$\geq \operatorname{ICDAS} 4$ & $50.0(16)$ & $46.7(14)$ & $0.893^{\dagger}$ \\
\hline ICDAS 0-3 & $50.0(522)$ & $48.4(15)$ & \\
\hline All included teeth & $n=475$ & $n=514$ & \\
\hline$\geq \operatorname{ICDAS} 4$ & $7.8(37)$ & $8.0(473)$ & $0.913^{\ddagger}$ \\
\hline ICDAS 0-3 & $92.2(438)$ & $92.0(41)$ & \\
\hline
\end{tabular}

ICDAS, International Caries Detection and Assessment System; $\mathrm{NaFV}$, sodium fluoride varnish; GIS, glass ionomer sealant. ${ }^{\dagger} p$ values derived from $\chi^{2}$ statistics. ${ }^{\ddagger} p$ values derived from Fisher's exact statistics.

only baseline dmfs score and individual molar ICDAS code (code 3) were associated with an increased likelihood of caries progression to dentin. The other 7 demographic factors, 6 oral health-related habit factors, oral hygiene (baseline VPI score), and study intervention all had no statistically significant relationships with caries progression to dentin (ICDAS code 4) at 12 months (Table 5).

\section{Discussion}

To our knowledge, there is no published study that compared the efficacy of GIS and NaFV on primary molars $[(*)]$. The present study is the first randomized controlled trial with an adequate sample size that compared
Table 4. Six- and 12-month GIS retention rates of included primary second molars

\begin{tabular}{|c|c|c|}
\hline & $\begin{array}{l}6 \text { months } \\
\%(n)\end{array}$ & $\begin{array}{l}12 \text { months } \\
\%(n)\end{array}$ \\
\hline \multicolumn{3}{|l|}{ Tooth 55} \\
\hline Baseline $(n=141)$ & $n=131$ & $n=120$ \\
\hline Dislodged & $75.8(99)$ & $89.2(107)$ \\
\hline Partially retained & $20.5(27)$ & $10.0(12)$ \\
\hline Completely retained & $3.8(5)$ & $0.8(1)$ \\
\hline \multicolumn{3}{|l|}{ Tooth 65} \\
\hline Baseline $(n=143)$ & $n=135$ & $n=123$ \\
\hline Dislodged & $79.3(107)$ & $86.1(106)$ \\
\hline Partially retained & $14.1(19)$ & $13.0(16)$ \\
\hline Completely retained & $6.7(9)$ & $0.8(1)$ \\
\hline \multicolumn{3}{|l|}{ Tooth 75} \\
\hline Baseline $(n=151)$ & $n=143$ & $n=133$ \\
\hline Dislodged & $76.9(110)$ & $82.7(110)$ \\
\hline Partially retained & $14.7(21)$ & $12.0(16)$ \\
\hline Completely retained & $8.4(12)$ & $5.3(7)$ \\
\hline \multicolumn{3}{|l|}{ Tooth 85} \\
\hline Baseline $(n=159)$ & $n=148$ & $n=138$ \\
\hline Dislodged & $70.3(104)$ & $87.7(121)$ \\
\hline Partially retained & $25.0(37)$ & $10.9(15)$ \\
\hline Completely retained & $4.7(7)$ & $1.4(2)$ \\
\hline \multicolumn{3}{|l|}{ All included teeth } \\
\hline Baseline $(n=592)$ & $n=557$ & $n=514$ \\
\hline Dislodged & $75.4(420)$ & $86.4(446)$ \\
\hline Partially retained & $18.7(104)$ & $11.4(59)$ \\
\hline Completely retained & $5.9(33)$ & $2.1(11)$ \\
\hline
\end{tabular}

these 2 interventions. Findings of this clinical trial suggest no difference between NaFV and GIS regarding their effectiveness in preventing occlusal surface caries in primary second molars. Thus, the null hypothesis that there is no difference between the placement of GIS and topical application of $5 \% \mathrm{NaFV}$ in the prevention of occlusal caries in primary second molars among preschool children cannot be rejected. The current findings coincide with those identified among permanent molars, suggesting similar occlusal caries preventive effects for $\mathrm{NaFV}$ and GIS $[(*)]$.

Multiple clinical trials have suggested the inferiority of TFV in preventing occlusal caries in permanent molars compared with RBS, but the low internal validity among most trials has compromised the quality of evidence $\left[\left(^{*}\right)\right]$. Related evidence among primary molars is even more scarce, with only 2 trials suggesting potential merits of combining RBS placement with TFV in occlusal caries prevention compared with using TFV alone $[(*) ;(*)]$, 
Table 5. Relationship between caries progression to ICDAS code 4 and selected independent variables at 12 months

\begin{tabular}{lllll}
\hline Independent variable & Estimate & $\begin{array}{l}\text { SE } \\
\text { (estimate) }\end{array}$ & $p$ value & $\begin{array}{l}\text { Multiple } \\
\text { comparison }\end{array}$ \\
\hline $\begin{array}{l}\text { Child demographic profile } \\
\text { Baseline dmfs score }\end{array}$ & 1.11 & 0.04 & $0.005^{* *}$ & \\
$\begin{array}{l}\text { Baseline second molar occlusal ICDAS score } \\
\text { (1) ICDAS code 0-1 }\end{array}$ & 0.07 & 0.47 & $<0.001^{* * *}$ & $(1)<(3)$ \\
$\quad$ (2) ICDAS code 2 & 0.26 & 0.52 & & $(2)<(3)$ \\
$\quad$ (3) ICDAS code 3 & & & & \\
$\quad$ (Intercept) & & & $0.112^{\dagger}$ & \\
\hline
\end{tabular}

ICDAS, International Caries Detection and Assessment System; dmfs, decayed, missing, and filled primary surface. ${ }^{\dagger}$ Reference category. ${ }^{* *} p<0.01 .{ }^{* * *} p<0.001$. whereas no randomized controlled trials have compared the efficacies of any sealants with TFV $\left[\left(^{*}\right)\right]$.

In the current study, the retention rate of GIS was only $13.5 \%$ at 12 months, which was lower than those reported in other studies, around $20 \%$ among primary molars at 18 months $[(*)]$, whereas among permanent molars, the retention rate of GIS varied considerably from $1 \%$ to $84 \%$ $[(*)]$. The difficulty in placing GIS in preschool children with limited cooperation and placement in a compromised outreach setting without rubber dam and suction may have contributed to the relatively lower retention rate. Different viscosities of GIS may also affect the sealant performance, with high-viscosity GIS performed better than low-viscosity GIS when placed with a fingerpressed technique $[(*) ;(*)]$. A medium-viscosity GIS (Fuji VII) was chosen in the current study to compare the efficacies of GIS in general with NaFV. It would be important to have more investigations and comparisons between GIS with other viscosities and NaFV. Despite having a lower retention rate than RBS in permanent teeth $\left.\left[{ }^{*}\right)\right]$, the caries prevention effect of GIS is not inferior $[(*)]$. A proposed explanation for the caries-preventive effect of GIS, despite its low retention rate comes from laboratory study findings. In vitro studies have found remnants of GIS being microscopically retained, despite dislodgement of the main bulk of sealant material, which can constantly release fluoride ions and contribute to caries prevention $\left[\left(^{*}\right) ;(*)\right]$. However, further validation of whether microscopically retained GIS can provide a sustained caries-protective effect is required.

Another possible reason for the lack of significant difference between GIS and NaFV in preventing caries in the primary second molars in the present clinical trial is relatively short (12 months) follow-up time, precluding a significant difference to be detected over time. The incidence of dentin caries found in the present study was only
$7.9 \%$ at 12 months. This result is not surprising as enamel caries lesions, on average, require 0.8 years to progress to the outer half of dentin and a further 1.4 years to develop into the inner half $[(*)]$.

The regression analyses in the present study indicate only baseline dmfs score, and an individual molar ICDAS code had significant associations with the development of dentinal caries at 12 months. These findings are consistent with most caries prediction models for children and adults found in the literature, in which baseline caries experience has been demonstrated as one of the most powerful indicators of caries increment or future development of new caries $[(*)]$. The primary second molars presenting with localized enamel breakdown (ICDAS code 3 ) were also found more likely to develop dentin caries, and neither GIS nor TFV was able to completely arrest the process. In fact, GIS has been scarcely used in clinical practice and reported to arrest fissure caries in the literature. This might be attributed to the lower retention rate and increased microleakage of GIS as compared to RBS $\left[(*) ;\left(^{*}\right)\right]$. The latter being a different sealant material with a higher retention rate $\left[\left(^{*}\right)\right]$ was more commonly used and investigated for occlusal caries arrest [Griffin et al., $2008 ;(*)]$. A recent network meta-analysis found that the combined use of RBS and TFV was the most effective method to reverse noncavitated occlusal carious lesions when compared with other nonrestorative regimens $[(*)]$. Probably, a combination of different noninvasive and microinvasive treatments would be better than using a single intervention in the management of caries in primary molars.

In our study, socioeconomic factors and oral health habits were not found to have significant associations with the development of dentinal caries in primary second molars with prevention measures implemented. These factors have been reported as risk factors for future caries increment in 
a number of longitudinal studies $\left[\left(^{*}\right) ;(*)\right]$. A caries prediction model for Hong Kong preschool children suggested that night nursing bottle habit, parental education attainment, and snacking habit were all significantly associated with higher caries increment at 2 years $[(*)]$. However, these factors were not found to be associated with caries increment in this clinical trial. The difference might be due to the provision of preventive measures to all study participants or simply due to different durations of follow-up.

Limitations of the present study include the difficulty to achieve blinding of the outcome assessors due to the obvious appearance of pink GIS, if present. However, blinding was still possible in over $75 \%$ of the cases due to the relatively high dislodgement rate at both the 6-month and 12-month follow-ups. Also, further blinding was attempted with the follow-ups being performed by a different reviewer with no knowledge of the intervention allocation. Blinding of both parents and children might not be possible as the GIS could be easily identified. However, the deviation from usual practice due to the different interventions received was not likely, and hence not making a substantial impact on the outcome. It is noted that there may be a selection bias because a clinical examination was only conducted among subjects with parental consent obtained, whose parents might also be more motivated toward improving their children's oral health. Attrition bias with respect to loss to follow-up does not post a major concern to the present study as the proportions of subject absence and withdrawal were similar across the 2 groups. Also, since the dropout rate of the study participants was only $13.3 \%$, and with no significant difference found between the children lost to follow-up and those examined at 12 months, the influence of attrition is likely to be mild.

\section{Conclusion}

Quarterly NaFV application and single GIS placement showed similar effectiveness in the prevention of occlusal caries development among preschool children. Only baseline caries experience and the presence of incipient enamel caries on occlusal surfaces were significantly associated with caries progression in primary second molars.

\section{Acknowledgments}

We thank Ms. LI Kar Yan Samantha for her outstanding assistance in statistical analysis. We also appreciate all the parents and children who participated in the current study.

\section{Statement of Ethics}

The current study complies with the guidelines for human studies and was conducted ethically in accordance with the World Medical Association Declaration of Helsinki. Written parental consent was received from each participant. The study protocol was approved by the Institutional Review Board of the University of Hong Kong/Hospital Authority Hong Kong West Cluster (UW 18-053) with the trial registered in ClinicalTrials.gov (NCT04163354).

\section{Conflict of Interest Statement}

The authors declare no potential conflicts of interest concerning the authorship as well as the publication of this article.

\section{Funding Sources}

No specific grant was received for this study.

\section{Author Contributions}

L.P.P.Y., E.M., L.G.H.M., and Y.C.K.Y contributed to the study conception and design. Material preparation and data collection were carried out by L.P.P.Y., S.D., and L.W. The analysis was performed by L.P.P.Y., who also took the lead in writing the first draft of the manuscript. All authors commented on the previous versions of the manuscript. All authors read and approved the final manuscript.

\section{References}

Primary Molar Occlusal Caries: Sealant or Varnish?
Ahovuo-Saloranta A, Forss H, Hiiri A, Nordblad A, Mäkelä M. Pit and fissure sealants versus fluoride varnishes for preventing dental decay in the permanent teeth of children and adolescents. Cochrane Database Syst Rev. 2016 Jan;18(1):CD003067.

Ahovuo-Saloranta A, Forss H, Walsh T, Nordblad A, Mäkelä M, Worthington HV. Pit and fissure sealants for preventing dental decay in permanent teeth. Cochrane Database Syst Rev. 2017 Jul 31;7(7):CD001830.
Ainamo J, Bay I. Problems and proposals for recording gingivitis and plaque. Int Dent J. 1975 Dec;25(4):229-35.

American Academy of Pediatric Dentistry. Guideline on caries-risk assessment and management for infants, children, and adolescents. Pediatr Dent. 2016 Oct;38(6):142-9.

American Dental Association Council on Scientific Affairs. Professionally applied topical fluoride: evidence - based clinical recommendations. J Dent Educ. 2007 Mar;71(3):393-402. 
Antonson SA, Antonson DE, Brener S, Crutchfield J, Larumbe J, Michaud C, et al. Twentyfour month clinical evaluation of fissure sealants on partially erupted permanent first molars: glass ionomer versus resin-based sealant. J Am Dent Assoc. 2012 Feb;143(2):115-22.

Bakhshandeh A, Ekstrand K. Infiltration and sealing versus fluoride treatment of occlusal caries lesions in primary molar teeth. 2-3 years results. Int J Paediatr Dent. 2015 Jan;25(1): 43-50.

Butail A, Dua P, Mangla R, Saini S, Chauhan A, Rana S. Evaluation of marginal microleakage and depth of penetration of different materials used as pit and fissure sealants: an in vitro study. Int J Clin Pediatr Dent. 2020 Jan;13(1): $38-42$.

Cagetti MG, Carta G, Cocco F, Sale S, Congiu G, Mura A, et al. Effect of fluoridated sealants on adjacent tooth surfaces: a 30-mo randomized clinical trial. J Dent Res. 2014 Jul;93(7 Suppl): 59S-65S.

Duangthip D, Wong MCM, Chu CH, Lo ECM. Caries arrest by topical fluorides in preschool children: 30-month results. J Dent. 2018 Mar; 70:74-9.

Frencken J, Amerogen E, Phantumvanit P, Songpaisan Y, Pilot T. Manual for the Atraumatic Restorative Treatment approach to control dental caries. 3rd ed. WHO Collaborating Centre for Oral Health Services Research; 1997.

Gooch BF, Griffin SO, Gray SK, Kohn WG, Rozier RG, Siegal M, et al. Preventing dental caries through school-based sealant programs: updated recommendations and reviews of evidence. J Am Dent Assoc. 2009 Nov;140(11): 1356-65.
Griffin SO, Oong E, Kohn W, Vidakovic B, Gooch BF, Dental Sealant Systematic Review Work Group. The effectiveness of sealants in managing caries lesions. J Dent Res. 2008 Feb; 87(2):169-74.

Honkala S, ElSalhy M, Shyama M, Al-Mutawa SA, Boodai H, Honkala E. Sealant versus fluoride in primary molars of kindergarten children regularly receiving fluoride varnish: one-year randomized clinical trial follow-up. Caries Res. 2015;49(4):458-66.

Ismail AI, Sohn W, Tellez M, Amaya A, Sen A, Hasson $\mathrm{H}$, et al. The international caries detection and assessment system (ICDAS): an integrated system for measuring dental caries. Community Dent Oral Epidemiol. 2007 Jun; 35(3):170-8.

Lam PPY, Sardana D, Ekambaram M, Lee GHM, Yiu CKY. Effectiveness of pit and fissure sealants for preventing and arresting occlusal caries in primary molars: a systematic review and meta-analysis. J Evid Based Dent Pract. 2020 Jun;20(2):101404.

Luo W, Lee GHM, Wong GHM, Wong MCM. Oral Health Attitude and Knowledge of the Primary Caregivers of infants with High Caries Risk: IAPD20 virtual; 2020.

Marinho VC, Worthington HV, Walsh T, Clarkson JE. Fluoride varnishes for preventing dental caries in children and adolescents. Cochrane Database Syst Rev. 2013:Cd002279.

Mejare I, Mjor IA. Glass ionomer and resin-based fissure sealants: a clinical study. J Dent Res. 1990 Aug;98(4):345-50.
Mickenautsch S, Yengopal V. Caries-preventive effect of glass ionomer and resin-based fissure sealants on permanent teeth: an update of systematic review evidence. BMC Res Notes. 2011 Jan 28;4:22.

Mickenautsch S, Yengopal V. Caries-preventive effect of high-viscosity glass ionomer and resin-based fissure sealants on permanent teeth: a systematic review of clinical trials. PLoS One. 2016 Jan 22;11(1):e0146512.

Nunn JH, Murray JJ, Smallridge J. British society of paediatric dentistry: a policy document on fissure sealants in paediatric dentistry. Int $J$ Paediatr Dent. 2000 Jun;10(2):174-7.

Ovrebo RC, Raadal M. Microleakage in fissures sealed with resin or glass ionomer cement. Scand J Dent Res. 1990 Feb;98(1):66-9.

Splieth C, Förster M, Meyer G. Additional caries protection by sealing permanent first molars compared to fluoride varnish applications in children with low caries prevalence: 2 -year results. Eur J Paediatr Dent. 2001 Jan;2(3):1337.

Tagliaferro EP, Pereira AC, Meneghim MC, Ambrosano GM. Assessment of dental caries predictors in a seven-year longitudinal study. J Public Health Dent. 2006;66(3):169-73.

Urquhart O, Tampi MP, Pilcher L, Slayton RL, Araujo MWB, Fontana M, et al. Nonrestorative treatments for caries: systematic review and network meta-analysis. J Dent Res. 2019 Jan;98(1):14-26.

Wong MC, Lu HX, Lo EC. Caries increment over 2 years in preschool children: a life course approach. Int J Paediatr Dent. 2012 Mar;22(2): 77-84.

World Health Organization. Oral health surveys: basic methods. 5th ed. World Health Organization; 2013. 